\title{
Topological spin transport of photons: Magnetic monopole gauge field in Maxwell's equations and polarization splitting of rays in periodically inhomogeneous media
}

\author{
K. Yu. Bliokh ${ }^{1,2, *}$ and V. D. Freilikher ${ }^{2,3}$ \\ ${ }^{1}$ Institute of Radio Astronomy, 4 Krasnoznamyonnaya st., Kharkov, 61002 Ukraine \\ ${ }^{2}$ Department of Physics, Bar-Ilan University, Ramat Gan 52900, Israel \\ ${ }^{3}$ Complex Photonic Systems, Department of Science and Technology and MESA +Research Institute, University of Twente, \\ P.O. Box 217, 7500 AE Enschede, The Netherlands
}

(Received 19 May 2004; revised manuscript received 28 February 2005; published 7 July 2005)

\begin{abstract}
Topological spin transport of electromagnetic waves (photons) in stationary smoothly inhomogeneous isotropic medium is studied. By diagonalizing the photon kinetic energy in Maxwell equations, we derive the non-Abelian pure gauge potential in the momentum space, which in adiabatic approximation for transverse waves takes the form of two $\mathbf{U}(1)$ Abelian potentials corresponding to magnetic monopole-type fields. These fields act on circularly polarized waves resulting in the topological spin transport of photons. We deduce general semiclassical (geometrical optics) ray equations that take into account a Lorentz-type force of the magnetic-monopole-like gauge field. Detailed analysis of rays in three-dimensional medium with twodimensional periodic inhomogeneity is presented. It is shown that rays located initially in the inhomogeneity plane experience topological deflections or splitting that move them out from this plane. The dependence of the rays' deflection on the parameters of the medium and on the direction of propagation is studied.
\end{abstract}

DOI: 10.1103/PhysRevB.72.035108

PACS number(s): 42.15.-i, 41.20.Jb, 03.65.Vf, 05.60.-k

\section{INTRODUCTION}

The remarkable property of Maxwell equations is that, being the main tool for the description of the classical electromagnetic waves, they also represent the quantum relativistic Schrödinger-type equations for photons, where the permittivity of a (inhomogeneous) medium and wave polarization play the roles of the potential energy and photon spin, respectively. That is why investigating electromagnetic waves, one can discover some fundamental properties of relativistic quantum particles with a spin. For example, the geometrical phase introduced by Berry in the general form, ${ }^{1}$ had been first described theoretically by Rytov ${ }^{2}$ and Vladimirskii ${ }^{3}$ (see also Ref. 4) in terms of the polarization evolution of electromagnetic waves. Experimentally, the Berry phase was observed first also for electromagnetic waves. ${ }^{5}$ In what follows, we assume the equivalence of the notions "photon" and "electromagnetic wave" as well as their "spin" and "polarization," respectively.

In recent years, progressively increasing attention has been given to the phenomena of topological spin transport of quantum particles (see, for instance, Refs. 6-13 and references therein). These effects are closely related to the general notion of Berry phase and thus should also show up in electromagnetic wave transport. Indeed, one can find the first description of polarization transport phenomena in works by Zel'dovich et al., ${ }^{14,15}$ where the deflections of circularly polarized light beams (so-called "optical Magnus effect") had been predicted theoretically and observed experimentally in fibers. Later it was shown (Refs. 16-20) that this effect represents the topological spin transport of photons. The essence of the phenomenon is that in an inhomogeneous medium, a polarization (spin) current arises that is directed orthogonally to the local wave vector. It can lead to the topological splitting of a ray of mixed (noncircular) polariza- tion into two circularly polarized rays. As it was demonstrated in Refs. 18 and 19, this effect is completely analogous to the anomalous and spin Hall effects in solids (see Refs. $6-8,10,11$, and 13).

In this paper we present a rigorous derivation of the gauge field for electromagnetic waves (photons) directly from the Maxwell equations using the standard diagonalization procedure (see Refs. 7, 12, and 13). As the result, a U(3) nonAbelian pure gauge potential arises in the momentum space, which is reduced in the adiabatic approximation to $\mathbf{U}(2)$ $\times \mathbf{U}(1)$ nontrivial potential. Here $\mathbf{U}(2)$ potential corresponds to the transverse electromagnetic waves and takes the form of a product of Abelian gauge potentials $\mathbf{U}^{2}(1)=\mathbf{U}(1)$ $\times \mathbf{U}(1)$ with magnetic-monopole-type gauge fields of opposite signs. Thus, the initial three-dimensional (3D) vector equation breaks up into two scalar Helmholtz equations with additional gauge $\mathbf{U}(1)$ potentials in the momentum space. These equations describe two eigenstates of a photon with helicity \pm 1 (in other words, right-hand and left-hand circularly polarized electromagnetic waves), that undergoes topological spin deflections in inhomogeneous dielectric media. The adiabatic approximation used is equivalent (for stationary medium) to the semiclassical, or geometrical optics approximation; i.e., it implies smoothness of the medium. The effect of topological polarization deflection in a 3D medium with periodic two-dimensional (2D) inhomogeneity is studied. We show that right-hand and left-hand circularly polarized rays located in the inhomogeneity plane can be deflected in opposite directions orthogonally to this plane due to topological polarization transport. The deflection strongly depends on the angle of wave propagation, and for the square lattice has a sharp peak at the angle $\pi / 4$. For samples long enough, there are also additional sharp peaks that correspond to irrational values of the tangent of the angle. If the ray has mixed (noncircular) polarization, the topological polarization 
transport can lead to its splitting into two circular rays or to the depolarization.

\section{II. "MAGNETIC MONOPOLE" GAUGE FIELD IN MOMENTUM SPACE IN MAXWELL EQUATIONS}

\section{A. Diagonalization of Maxwell equations and appearance of a gauge potential}

Let us consider a monochromatic electromagnetic wave in a stationary isotropic inhomogeneous medium characterized by the refractive index $n(\mathbf{R})=\sqrt{\varepsilon(\mathbf{R})}$ ( $\varepsilon$ is the dielectric constant of the medium, and $\mathbf{R}$ is the coordinate vector). Maxwell equations for the wave electric field $\mathcal{E}$ have the form

$$
\left[\text { curl curl }-k_{0}^{2} n^{2}\right] \mathcal{E}=0,
$$

where $k_{0}=\omega / c$. If we introduce the dimensionless momentum differential operator

$$
\mathbf{p}=-i k_{0}^{-1} \frac{\partial}{\partial \mathbf{R}},
$$

Eq. (1) takes the form

$$
-\mathbf{p} \times(\mathbf{p} \times \mathcal{E})-n^{2} \mathcal{E}=0 .
$$

(For monochromatic electromagnetic waves and Maxwell equations, it is more convenient to define the momentum with factor $k_{0}^{-1}$ rather than with Planck's constant $\hbar$.)

Equation (3) is a relativistic Schrödinger-type equation

$$
\hat{H} \mathcal{E}=0,
$$

with matrix-valued Hamiltonian differential operator (here and below we mark matrices by hats)

$$
\hat{H}(\mathbf{p}, \mathbf{R})=\hat{I}\left[p^{2}-n^{2}(\mathbf{R})\right]-\hat{Q} .
$$

Here $Q_{i j}=p_{i} p_{j}, \hat{I}$ is $3 \times 3$ unit matrix, $p=|\mathbf{p}|$, and $-n^{2}$ and $\hat{I} p^{2}-\hat{Q}$ play the roles of the potential energy and nondiagonal kinetic energy of the electromagnetic wave (photons), respectively. Note that the photon Hamiltonian operator in quantum electrodynamics is quite similar to Eq. (5) and has the same nondiagonal part. ${ }^{21}$

Obviously, nondiagonal Hamiltonian (5) mixes up different independent eigenstates of the wave. To find these eigenstates and to describe their evolution, we diagonalize the Hermitian Hamiltonian (5) using the general procedure with an unitary matrix $\hat{U}(\mathbf{p})$ (see, for instance, Refs. 7, 12, and 13). Matrix $\hat{U}$ is built of the orthogonal set of unit eigenvectors of matrix $\hat{H}$ and can be chosen as

$$
\hat{U}=\left(\begin{array}{ccc}
\frac{p_{y}}{\sqrt{p_{x}^{2}+p_{y}^{2}}} & -\frac{p_{x} p_{z}}{p \sqrt{p_{x}^{2}+p_{y}^{2}}} & \frac{p_{x}}{p} \\
-\frac{p_{x}}{\sqrt{p_{x}^{2}+p_{y}^{2}}} & -\frac{p_{y} p_{z}}{p \sqrt{p_{x}^{2}+p_{y}^{2}}} & \frac{p_{y}}{p} \\
0 & \frac{\sqrt{p_{x}^{2}+p_{y}^{2}}}{p} & \frac{p_{z}}{p}
\end{array}\right) .
$$

With similarity transformation with Eq. (6), the nondiagonal part $\hat{Q}$ of Hamiltonian (5) becomes diagonal:

$$
\hat{U}^{-1} \hat{Q} \hat{U}=\hat{\Lambda} \equiv \operatorname{diag}\left(\lambda_{1}, \lambda_{2}, \lambda_{3}\right)=\left(\begin{array}{ccc}
0 & 0 & 0 \\
0 & 0 & 0 \\
0 & 0 & p^{2}
\end{array}\right)
$$

One can see that two eigenvalues of $\hat{Q}$ coincide with each other and are equal to zero, $\lambda_{1,2}=0$, while the third one is equal to $\lambda_{3}=p^{2}$. It follows from Eqs. (5) and (7) that eigenvalues $\lambda_{1,2}$ correspond to transverse electromagnetic waves with local dispersion law $p^{2}=k_{0}^{2} n^{2}$ (or $\omega^{2}=k^{2} c^{2}$, where $k$ is the local wave number), while the eigenvalue $\lambda_{3}$ corresponds to the longitudinal wave, which can exist only at resonances where $n=0 .{ }^{22}$ In what follows, we assume that such resonant points (if they exist) are well off the region of propagation, and longitudinal waves are not excited. The transverse wave state is double degenerated that represents a polarization degeneration of electromagnetic waves: a phenomenon well known in geometrical optics of isotropic media, ${ }^{22}$ and corresponds to the spin degeneration of energy levels of relativistic quantum particles. Note also that unitary transformation with $\hat{U}(\mathbf{p})$ is equivalent to the local rotation of the coordinate frame in such a way that the $z$ axis in each point is directed along $\mathbf{p}$ [it can be seen from the comparison of Eq. (7) with the initial matrix $\left.Q_{i j}=p_{i} p_{j}\right]$. Therefore, in the new coordinate frame the electric field $\mathbf{E}$ of the longitudinal state is directed practically along the $z$ axis, while the electric field of the transverse states lies almost in $(x, y)$ plane (see below).

Transformation (6) being applied to Eq. (4) with Hamiltonian (5) after the substitution $\mathcal{E}=\hat{U} \mathbf{E}$ yields:

$$
\left\lfloor\hat{I} p^{2}-\hat{\Lambda}-\hat{U}^{-1} n^{2} \hat{U}\right\rfloor \mathbf{E}=0 .
$$

If momentum $\mathbf{p}$ and coordinates $\mathbf{r}$ are ordinary commuting numbers, the potential part of the Hamiltonian (8) is a scalar: $n^{2}$. However, when electromagnetic wave (or quantum particle) is concerned, the momentum and coordinates are noncommuting quantities that can be represented by differential operators. Therefore, in $\mathbf{p}$ representation in Eq. (8), we have

$$
\begin{aligned}
\hat{U}^{-1}(\mathbf{p}) n^{2}(\mathbf{R}) \hat{U}(\mathbf{p}) & =\hat{U}^{-1}(\mathbf{p}) n^{2}\left(i k_{0}^{-1} \frac{\partial}{\partial \mathbf{p}}\right) \hat{U}(\mathbf{p}) \\
& =n^{2}\left(i k_{0}^{-1} \hat{I} \frac{\partial}{\partial \mathbf{p}}+k_{0}^{-1} \hat{\mathbf{A}}\right) \\
& =n^{2}\left(\hat{I} \mathbf{R}+k_{0}^{-1} \hat{\mathbf{A}}\right),
\end{aligned}
$$

where

$$
\hat{\mathbf{A}}(\mathbf{p})=i \hat{U}^{-1} \frac{\partial \hat{U}}{\partial \mathbf{p}}
$$

is a pure gauge non-Abelian potential induced in the momentum p space by the local gauge transformation $\hat{U} \in \mathbf{U}(3){ }^{7,12,13}$ In accordance to the gauge field theory and Hamiltonian mechanics, in Eq. (9) $\mathbf{R}$ are canonical (or generalized in classical mechanics) coordinates that correspond to the "usual" derivatives $i k_{0}^{-1} \partial / \partial \mathbf{p}$. At the same time, $o b$ servable (i.e., related to "the center of the particle") coordinates $\hat{\mathbf{r}}$ correspond now to the covariant derivatives: 


$$
\hat{\mathbf{r}}=i k_{0}^{-1} \frac{D}{D \mathbf{p}}=i k_{0}^{-1} \hat{I} \frac{\partial}{\partial \mathbf{p}}+k_{0}^{-1} \hat{\mathbf{A}} \text {. }
$$

Matrix-valued coordinates $\hat{r}_{i}$ commute with each other, $\left[\hat{r}_{i}, \hat{r}_{j}\right]=0$, because the potential (10) is a pure gauge one, and corresponding field tensor is zero (see Refs. 7 and 13).

Thus, Eq. (8) after substitutions from Eqs. (9) and (10) takes the form

$$
\left[\hat{I} p^{2}-\hat{\Lambda}-n^{2}\left(\hat{I} \mathbf{R}+k_{0}^{-1} \hat{\mathbf{A}}\right)\right] \mathbf{E}=0 .
$$

Although this equation has a Hamiltonian of a diagonal form, the potential energy $n^{2}$ contains a nondiagonal gauge vector potential $\hat{\mathbf{A}}$. This potential describes all nontrivial (i.e., those that are not determined by the current eigenvalues of the initial Hamiltonian) evolutions of the different polarization states of the wave. Direct calculation of Eq. (10) with Eq. (6) leads to the following expressions for the Hermitian antisymmetric matrices $\hat{A}_{p_{i}}$ :

$$
\hat{A}_{p_{x}}=i\left(\begin{array}{ccc}
0 & -\frac{p_{y} p_{z}}{p\left(p_{x}^{2}+p_{y}^{2}\right)} & \frac{p_{y}}{p \sqrt{p_{x}^{2}+p_{y}^{2}}} \\
\frac{p_{y} p_{z}}{p\left(p_{x}^{2}+p_{y}^{2}\right)} & 0 & -\frac{p_{x} p_{z}}{p^{2} \sqrt{p_{x}^{2}+p_{y}^{2}}} \\
-\frac{p_{y}}{p \sqrt{p_{x}^{2}+p_{y}^{2}}} & \frac{p_{x} p_{z}}{p^{2} \sqrt{p_{x}^{2}+p_{y}^{2}}} & 0
\end{array}\right),
$$

In these expressions, $2 \times 2$ sectors that consist of $11,12,21$, and 22 elements describe the evolution of the double degenerated transverse states; the zero component 33 acts on the longitudinal state, while the cross-components 13, 23 and 31, 32 describe the transitions between transverse and longitudinal wave states.

\section{B. Adiabatic reduction and separation of eigenstates}

As was mentioned above, if the resonance points where $n(\mathbf{r})=0$ are far away from the region of propagation, longitudinal waves are not excited. Thus, in the smoothly inho- mogeneous medium the electric field is almost transverse $\left[\mathbf{E} \cong\left(E_{x}, E_{y}, 0\right)\right]$, and the longitudinal component of the electric field $E_{z}$ is proportional to the small geometrical optics (semiclassical) parameter $^{22}$

$$
\mu=\frac{1}{n k_{0} L} \ll 1
$$

where $L$ is the characteristic scale of the medium's inhomogeneity. Of the same order $\mu$ are the cross-state terms, which appear in the motion equations from the matrix operator (13). It is known that in this case their contribution to the state's evolution is proportional to $\mu^{2}$ (see, for example in Ref. 23), and, therefore, in the first approximation in $\mu$ one should neglect 13, 23 and 31,32 components of $\hat{\mathbf{A}}$. The initial gauge potential $\mathbf{U}(3)$ is then reduced to $\mathbf{U}(2) \times \mathbf{U}(1)$, which corresponds to the adiabatic approximation (see, for instance, Refs. 7, 12, 13, and 20). [As is shown in Ref. 13, the adiabaticity condition for a nonlocalized (propagating) particle with stationary Hamiltonian is equivalent to a semiclassical condition similar to Eq. (14).] Thus, the transverse and longitudinal wave states become independent, and Eq. (12) breaks down into $2 \times 2$ matrix equation for the transverse states and a scalar equation for the longitudinal one. Matrix equation for two-dimensional transverse electric vector field $\widetilde{\mathbf{E}}=\left(E_{x}, E_{y}\right)$ has the form

$$
\left[\hat{I} p^{2}-n^{2}\left(\hat{I} \mathbf{R}+k_{0}^{-1} \hat{\mathbf{A}}^{(t r)}\right)\right] \widetilde{\mathbf{E}}=0
$$

Here $\hat{I}$ is $2 \times 2$ unit matrix; $\hat{\mathbf{A}}^{(t r)}$ denotes the "transverse" 2 $\times 2$ sector of the corresponding $3 \times 3$ matrices Eq. (13). In the adiabatic approximation $\hat{\Lambda}^{(t r)}=0$, and it disappears at conversion of Eq. (12) into Eq. (15).

As is well known from the Berry phase and spin gauge field theories, the potential $\hat{\mathbf{A}}^{(t r)}$, which corresponds to double degenerated state, is a $\mathbf{U}(2)$ non-Abelian gauge potential, which can be decomposed into full set of noncommuting Pauli matrices (see, for instance, Refs. 20, 24, and 25). However, as one can see from the explicit form of matrices (13), when it comes to photons, matrix $\hat{\mathbf{A}}^{(t r)}$ is proportional to the single Pauli matrix $\hat{\sigma}_{2}$ :

$$
\hat{\mathbf{A}}^{(t r)}=\left(\frac{p_{y} p_{z}}{p\left(p_{x}^{2}+p_{y}^{2}\right)},-\frac{p_{x} p_{z}}{p\left(p_{x}^{2}+p_{y}^{2}\right)}, 0\right) \hat{\sigma}_{2}, \quad \hat{\sigma}_{2}=\left(\begin{array}{cc}
0 & -i \\
i & 0
\end{array}\right) .
$$

Components of $\hat{\mathbf{A}}^{(t r)}$ commute with each other, which means that $\hat{\mathbf{A}}^{(t r)}$ represents the Abelian potential $\mathbf{U}^{2}(1)=\mathbf{U}(1)$ $\times \mathbf{U}(1)$. Obviously, we can rotate the reference frame in such a way that $\hat{\sigma}_{2}$ transforms into

$$
\hat{\sigma}_{3}=\left(\begin{array}{cc}
1 & 0 \\
0 & -1
\end{array}\right)
$$

and potential $\hat{\mathbf{A}}^{(t r)}$ becomes diagonal. Such uniform rotation is executed by the global substitution $\widetilde{\mathbf{E}}=\hat{V}^{(t r)} \boldsymbol{\Psi}$ with 


$$
\hat{V}^{(t r)}=\frac{1}{\sqrt{2}}\left(\begin{array}{cc}
1 & 1 \\
i & -i
\end{array}\right),
$$

which diagonalizes Eq. (15), and therefore breaks it down into two independent scalar equations:

$$
H^{ \pm} \Psi^{ \pm}=0, \quad H^{ \pm}=\frac{1}{2}\left[p^{2}-n^{2}\left(\mathbf{R}+k_{0}^{-1} \mathbf{A}^{ \pm}\right)\right]=\frac{1}{2}\left[p^{2}-n^{2}\left(\mathbf{r}^{ \pm}\right)\right],
$$

where $\mathbf{r}^{ \pm}=i k_{0}^{-1} \partial / \partial \mathbf{p}+k_{0}^{-1} \mathbf{A}^{ \pm}$are the operators of the coordinates of "the photon's center" (covariant coordinates) analogously to Eq. (11). Here we denote $\boldsymbol{\Psi} \equiv\left(\Psi^{+}, \Psi^{-}\right)$, a factor of $1 / 2$ is introduced for convenience, and $\mathbf{U}(1)$ gauge potentials are equal to

$$
\mathbf{A}^{ \pm}= \pm\left(\frac{p_{y} p_{z}}{p\left(p_{x}^{2}+p_{y}^{2}\right)},-\frac{p_{x} p_{z}}{p\left(p_{x}^{2}+p_{y}^{2}\right)}, 0\right) .
$$

Thus, we have reduced the initial vector problem of the evolution of a polarized wave to two independent scalar equations (18) with additional gauge potentials (19) (compare with scalar and vector geometric optics in Ref. 22).

It is worth noticing that two functions, $\Psi^{ \pm}=E_{x} \mp i E_{y}[$ see Eq. (17)] represent the basis of circularly polarized waves. This means that only circularly polarized states are the independent eigenstates of transverse electromagnetic wave. ${ }^{16-18}$ It is quite natural because these solutions are the photon spin eigenstates with helicities $\pm 1 .^{21}$ Of course we could apply the transformation (17) directly to the 3D Eq. (12). In this case, transformation matrix $\hat{V}$ is equal to

$$
\hat{V}=\frac{1}{\sqrt{2}}\left(\begin{array}{ccc}
1 & 1 & 0 \\
i & -i & 0 \\
0 & 0 & \sqrt{2}
\end{array}\right) .
$$

One can see that columns of this matrix are the eigenvectors of the $z$ component of the photon spin operator $\hat{s}_{3}{ }^{21}$ This fact supports our conclusion that functions $\Psi^{ \pm}=E_{x} \mp i E_{y}$ correspond to the independent photon spin states.

\section{Monopole type field in the momentum space and noncommuting coordinates}

After $\mathbf{U}(1)$ Abelian gauge potentials have been found [Eq. (19)], the corresponding field tensors (Berry curvatures) can be calculated directly, which yields

$$
F_{i j}^{ \pm}=\frac{\partial A_{j}^{ \pm}}{\partial p_{i}}-\frac{\partial A_{i}^{ \pm}}{\partial p_{j}}=\mp e_{i j k} \frac{p_{k}}{p^{3}},
$$

where $e_{i j k}$ is unit antisymmetric tensor. Equation (21) presents the magnetic-monopole-like field, although not in the real but in the momentum space (see, for example, Refs. 1, 4, $7,8,12,13,18-20$, and 26). Since $\mathbf{p}$ space is a 3D one, the field (21) can be represented as a pseudovector:

$$
\mathbf{F}^{ \pm}=\mp \frac{\mathbf{p}}{p^{3}}
$$

Clearly, these fields act (in $\mathbf{p}$ space) on the different spin (polarization) eigenstates of the photon $\left(\Psi^{ \pm}\right)$in opposite di- rections. As is known, such fields cause spin current of particles orthogonal to their motion. ${ }^{6-8,10-13,18-20}$ When photons are concerned, they lead to the topological splitting of right and left circularly polarized beams (photons). ${ }^{16-20}$

In a recent paper, ${ }^{20}$ non-Abelian spin gauge potential and field have been derived for a relativistic particle with arbitrary spin. For massless particles this field has the magneticmonopole-type configuration, which is characteristic for Abelian U(1) field. As we have shown above for photons, non-Abelian gauge field $\mathbf{U}(2)$ is indeed decomposed into two independent Abelian U(1) fields.

From Eq. (11) with potentials (19) and fields (21) and (22), it follows that coordinates of circularly polarized waves do not commute (see Refs. 7, 13, and 20):

$$
\left[r_{i}^{ \pm}, r_{j}^{ \pm}\right]=i k_{0}^{-2} F_{i j}^{ \pm}=\mp i k_{0}^{-2} e_{i j k} \frac{p_{k}}{p^{3}} .
$$

It is important to emphasize that nonzero field tensors (21) and commutation relations appear owing to the adiabatic reduction $\mathbf{U}(3) \rightarrow \mathbf{U}(2) \times \mathbf{U}(1)$ in the gauge potential (10) and (13). In the general $\mathbf{U}(3)$ case, potential (10) and (13) is a pure gauge one, and the field tensor, as well as the coordinate commutators, are zero. ${ }^{7,13}$

\section{Semiclassical ray equations, topological spin transport of photons, and Berry phase}

If the variations of the refractive index are smooth enough, so that the semiclassical condition (14) is satisfied, Hamiltonian equations of motion for photons (ray equations) can be derived in geometrical optics approximation, ${ }^{22}$ and have the form

$$
\dot{\mathbf{p}}=-\frac{\partial H^{ \pm}}{\partial \mathbf{R}}, \quad \dot{\mathbf{R}}=\frac{\partial H^{ \pm}}{\partial \mathbf{p}}
$$

or

$$
\dot{\mathbf{p}}=-\frac{\partial H^{ \pm}}{\partial \mathbf{r}^{ \pm}}, \quad \dot{\mathbf{r}}^{ \pm}=\frac{\partial H^{ \pm}}{\partial \mathbf{p}}+k_{0}^{-1}\left(\mathbf{F}^{ \pm} \times \dot{\mathbf{p}}\right),
$$

where dot denotes the derivative with respect to the ray parameter $s$, which is connected with the ray length $l$, as $d l$ $=n d s$. From now on, momentum $\mathbf{p}$ is a dimensionless wave vector (not a differential operator as above), $\mathbf{p}=k_{0}^{-1} \mathbf{k}$, while $\mathbf{r}^{ \pm}$are the coordinates on the rays of circular polarizations. At that, the dispersion relation $p=n$ is valid. Upon substituting Hamiltonian (18) into Eqs. (24), we obtain ${ }^{16-20}$

$$
\dot{\mathbf{p}}=\frac{1}{2} \frac{\partial n^{2}}{\partial \mathbf{r}^{ \pm}}, \quad \dot{\mathbf{r}}^{ \pm}=\mathbf{p} \mp k_{0}^{-1}\left(\frac{\mathbf{p}}{p^{3}} \times \dot{\mathbf{p}}\right) \text {. }
$$

Equations (25) are transformed into standard geometrical optics equations ${ }^{22}$ if the term proportional to $k_{0}^{-1}$ is neglected. This term represents a Lorentz-type force of the magnetic monopole-like field (22) in momentum space, which causes additional deflections of circularly polarized rays: $\mathbf{r}^{ \pm}=\mathbf{r}$ $+\delta \mathbf{r}^{ \pm}$, where $\mathbf{r}$ is the coordinates on the zero-approximation ray. These deflections can be calculated by the integration of the last term in second Eq. (25) and is presented as the contour integral ${ }^{16-18}$ 


$$
\delta \mathbf{r}^{ \pm}=\mp k_{0}^{-1} \int_{C} \frac{\mathbf{p} \times d \mathbf{p}}{p^{3}}
$$

where contour $C$ is the wave trajectory in the momentum space.

Lorentz-type force similar to that in Eq. (25) also arises at the nonrelativistic electron motion with spin-orbit interaction, where it leads to the anomalous and intrinsic spin Hall effects. ${ }^{6-8,10-13}$ In our case, the force splits the trajectories of right and left circularly polarized waves and, hence, induces a photon spin current orthogonal to the vector $\mathbf{p}$. Thus, the term that is proportional to $k_{0}^{-1}$ in Eqs. (25) and (26) presents the topological spin transport of photons, which can be associated with intrinsic spin Hall effect for photons (see Refs. 18 and 19). One of the manifestations of the Lorentz-type force in Eq. (25), the so-called optical Magnus effect, had been discovered in Refs. 14 and 15. Recently, Eqs. (25) have been derived and analyzed in details in terms of geometrical optics and Berry phase formalism in Refs. 16 and 17, and by postulating a monopole field (21) and (22) in geometrical optics equations in Refs. 18 and 19. In the present paper we present a rigorous derivation of the monopole gauge field (21) and (22) and transport Eqs. (25) directly from Maxwell equations.

Note that the Berry phase can also be deduced from the approach suggested above (see Refs. 13 and 18). Indeed, the semiclassical phase is determined in the canonical (generalized) coordinates, and for circularly polarized waves equals

$$
\begin{aligned}
\varphi^{ \pm} & =k_{0} \int_{0}^{\mathbf{R}} \mathbf{p} d \mathbf{R} \\
& =k_{0} \int_{0}^{\mathbf{r}^{ \pm}} \mathbf{p} d \mathbf{r}^{ \pm}-\left.\mathbf{p} \mathbf{A}^{ \pm}\right|_{\mathbf{p}_{0}} ^{\mathbf{p}}+\int_{C} \mathbf{A}^{ \pm} d \mathbf{p} \\
& =k_{0} \int_{0}^{\mathbf{r}} \mathbf{p} d \mathbf{r}+\int_{C} \mathbf{A}^{ \pm} d \mathbf{p} .
\end{aligned}
$$

Here the connection between observable and generalized coordinates [Eq. (18)], as well as the relations $\delta \dot{\mathbf{r}}^{ \pm} \perp \mathbf{p}$ [Eqs. (25) and (26)], and $\mathbf{A}^{ \pm} \perp \mathbf{p}$ [Eq. (19)], have been used; $\mathbf{p}_{0}$ is the initial momentum, and term $-\omega t$ in the phase is omitted. The first term in Eq. (27) is the ordinary dynamic phase. The second term is the Berry phase, which for closed contours (loops) can be presented as the flux of the field (21) and (22) through the surface $S$, which is stretched over the loop $C$ in p space:

$$
\theta_{B}=\oint_{C} \mathbf{A}^{ \pm} d \mathbf{p}=\int_{S} \mathbf{F}^{ \pm} d \mathbf{s}
$$

For noncircularly polarized electromagnetic waves, the Berry phase leads to the rotation of the polarization plane described theoretically in Refs. 2 and 3 (see also Ref. 4) and observed experimentally in Ref. 5.

\section{TOPOLOGICAL POLARIZATION SPLITTING OF RAYS IN A PERIODICALLY INHOMOGENEOUS MEDIUM}

As an example of the effects described above, we consider the deflection of rays in a two-dimensionally periodic medium. An advantage of this system is that being relatively simple for analytical analysis, it enables one to demonstrate the most general features of the topological polarization (spin) splitting in inhomogeneous media. On the other hand, 2D periodic systems are interesting by themselves because they find ever increasing applications in optronics and nanotechnology (photonic crystals, nanostructured dielectrics, etc.). Note that in one-dimensionally inhomogeneous media, where all rays are flat curves, the topological effects such as Berry phase and polarization splitting of rays (26) never occur. ${ }^{16,17}$

Let us assume that the refractive index of the medium of propagation is given by

$$
n^{2}(\mathbf{r})=n_{0}^{2}+n_{1}^{2}[\cos (\chi x)+\sin (\chi y+\beta)],
$$

where $n_{1}^{2} \ll n_{0}^{2}$ and $\beta \in(0,2 \pi)$ is a constant parameter. From now on we assume that parameters of the system are away from the resonance ones, and geometrical optics and perturbation methods are applicable. The approximations in use are certainly applicable when the sample is not too long, and the backscattering is negligible. The condition of validity of the geometrical optics approximation Eq. (14) means in the case Eq. (29) that the period of the medium's inhomogeneity should be much larger than the wavelength of the radiation:

$$
\frac{\chi}{n_{0} k_{0}} \ll 1
$$

To calculate ray deflections (26), we first find the "zeroapproximation" ray trajectory, which is determined by geometrical optics equations (25) with the last term in the second equation omitted. Substitution of Eq. (29) in (25) yields

$$
\dot{\mathbf{p}}=\frac{n_{1}^{2} \chi}{2}[-\sin (\chi x), \cos (\chi y+\beta), 0], \quad \dot{\mathbf{r}}=\mathbf{p} .
$$

We solve this system of nonlinear differential equations by the perturbation method under assumption that the variations of the refractive index are small in amplitude:

$$
\delta=\frac{n_{1}^{2}}{n_{0}^{2}} \ll 1 .
$$

If in zero-order approximation in $\delta$ the ray has the momentum (wave vector) $\mathbf{p}_{0}=\left(p_{x 0}, p_{y 0}, p_{z 0}\right)$ and crosses the origin $\mathbf{r}=0$, then in the first-order approximation in $\delta$ the solution of Eq. (31) gives the following trajectory of the wave in $\mathbf{p}$ space:

$$
\begin{gathered}
p_{x}=p_{x 0}+\frac{n_{1}^{2}}{2 p_{x 0}} \cos \left(\chi p_{x 0} s\right), \quad p_{y}=p_{y 0}+\frac{n_{1}^{2}}{2 p_{y 0}} \sin \left(\chi p_{y 0} s+\beta\right), \\
p_{z}=p_{z 0} .
\end{gathered}
$$

Deflections of circularly polarized rays can be found directly from Eq. (26) by substituting (33) and subsequent integration. It also can be estimated using rather convenient 

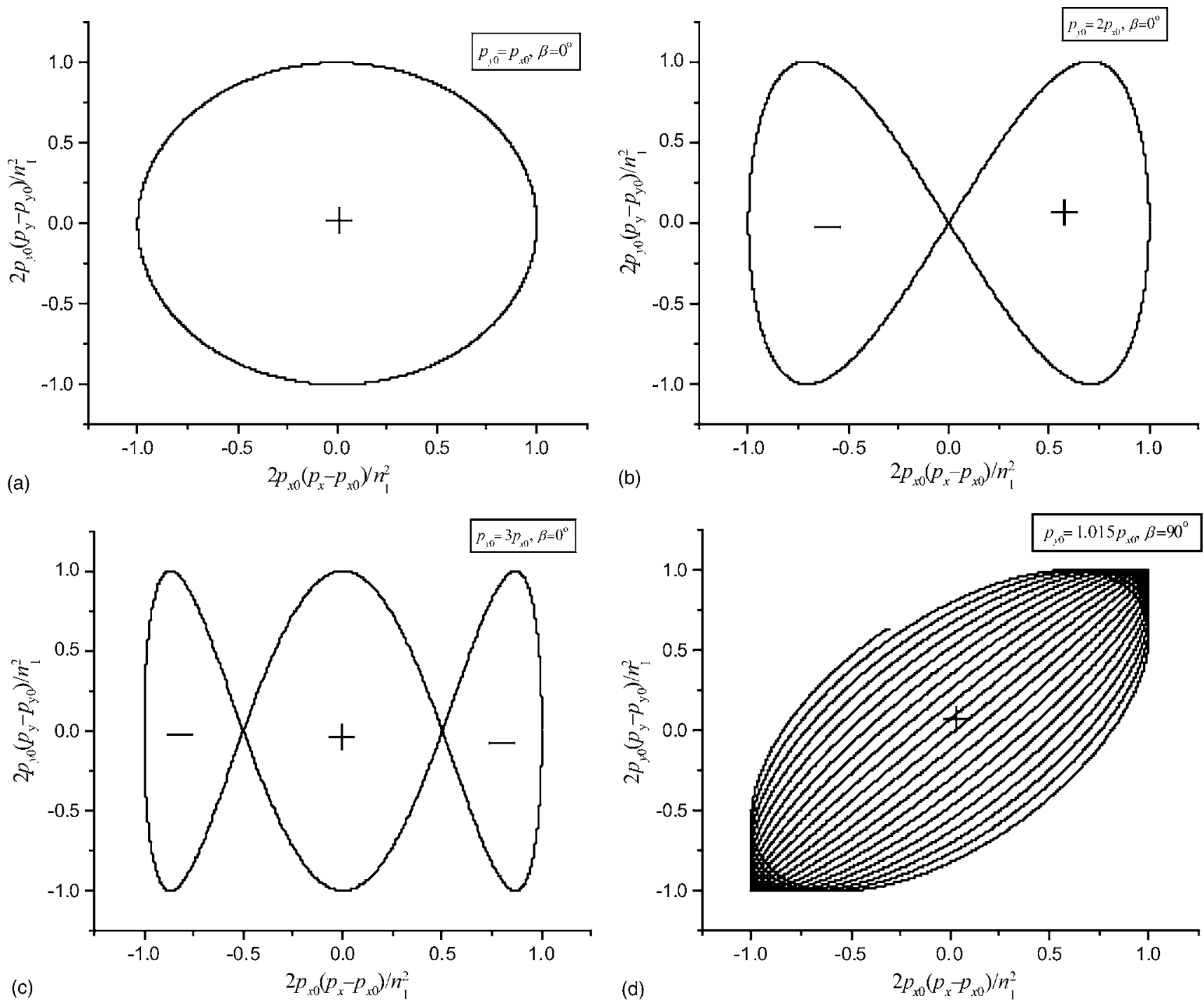

FIG. 1. Wave trajectories in the $\left(p_{x}, p_{y}\right)$ plane in the momentum space. Dimensionless units are used [see Eqs. (33)]. The signs of oriented areas of the loops are shown.

geometrical method proposed in Ref. 17, where it was shown that, in the case of closed in $\mathbf{p}$ space trajectories, the deviation of the circularly polarized ray is equal to the $\mathbf{p}$ gradient of the Berry phase; i.e.,

$$
\delta \mathbf{r}^{ \pm}=-k_{0}^{-1} \frac{\partial \theta_{B}}{\partial \mathbf{p}} .
$$

The Berry phase grows with the ray length, and its increment during one period of the cyclic evolution in $\mathbf{p}$ space is equal to the flux of the field $\mathbf{F}^{ \pm}$through contour $C$ [Eqs. (21), (22), and (28)]; i.e., to the solid angle $\Omega$ (with the corresponding sign), at which contour $C$ is seen from the origin of $\mathbf{p}$ space [see Eq. (28) and Refs. 1 and 4].

Let us analyze the rays located (in zero geometrical optics approximation) in the $(x, y)$ plane. To do this we assume that $p_{z 0} \rightarrow 0$ is infinitely small value [it is necessary for differentiation in Eq. (34)], and introduce the angle of propagation $\alpha$ as the angle between $\mathbf{p}_{0}$ and the $x$ axis:

$$
\tan \alpha=\frac{p_{y 0}}{p_{x 0}} .
$$

If the angle $\alpha=\pi / 4$, i.e., $p_{x 0}=p_{y 0}=p_{0} / \sqrt{2}$, according to Eq. (33) the trajectory $C$ in momentum space is a circle in the $\left(p_{x}, p_{y}\right)$ plane with the center at the point $\mathbf{p}_{0}$ and radius $n_{1}^{2} / \sqrt{2} p_{0}$ [Fig. 1(a)]. [So far we have assumed that $\beta=0$ in Eq. (33)]. At infinitely small $p_{z 0}$, this circle is seen from the origin of $\mathbf{p}$ space as an ellipse with the semi-axes $\rho_{1}$ $=n_{1}^{2} / \sqrt{2} n_{0}$ and $\rho_{2}=n_{1}^{2} p_{z 0} / \sqrt{2} n_{0}^{2}$. The area of this ellipse equals $\pi \rho_{1} \rho_{2}=n_{1}^{4} p_{z 0} / 2 n_{0}^{3}$, while the solid angle equals $\Omega$ $=\pi n_{1}^{4} p_{z 0} / 2 n_{0}^{5}$. Hence, the Berry phase per one period of the inhomogeneity is equal to

$$
\theta_{B 0}=\mp \frac{\pi n_{1}^{4} p_{z 0}}{2 n_{0}^{5}} .
$$

In one period the ray runs the length $l_{0}=2 \sqrt{2} \pi / \chi$, and after a number of periods (when the deflections become noticeable) Berry phase becomes approximately 


$$
\theta_{B} \approx \theta_{B 0} \frac{l}{l_{0}}=\mp \frac{n_{1}^{4} \chi p_{z 0}}{4 \sqrt{2} n_{0}^{5}} l,
$$

where $l$ is the total length of the ray. By substituting Eq. (37) into Eq. (34), we obtain

$$
\delta z^{ \pm} \approx \pm \frac{k_{0}^{-1} \chi n_{1}^{4}}{4 \sqrt{2} n_{0}^{5}} l .
$$

Note, that in spite of the fact that the Berry phase [Eqs. (36) and (37)] equals zero at $p_{z 0}=0$, their momentum gradient [Eqs. (34) and (38)], is a finite quantity (compare with examples in Ref. 17).

Equation (38) means that right-hand and left-hand circularly polarized rays propagating in the $(x, y)$ plane at $\alpha$ $=\pi / 4$ are deflected in the opposite $z$ directions; i.e., the polarization topological transport of electromagnetic waves in periodical medium [Eq. (29)] takes place. If electromagnetic wave has mixed polarization, the ray will split into two circularly polarized eigenrays. ${ }^{16-18}$ The effect, which is of the order of the small parameter $k_{0}^{-1} \chi$, is nevertheless proportional to length $l$ of the trajectory, and therefore can be significant at large distances.

It should be noted that the two-dimensionally inhomogeneous medium under consideration possesses TE and TM exact eigenmodes. However, linear birefringence of the TE and TM modes of the system is proportional to the second power of the small geometrical optics parameter (14) (see, for instance, Ref. 27), and thus should be neglected in the approximation in use. We emphasize also that the reflection symmetry of the medium and of the TE and TM eigenmodes with respect to the $z=0$ plane does not contradict to the deflections of the rays in the $z$ direction. The issue is that there is no transverse transport of the eigenmodes. However, their interference in the course of ray propagation leads to the effective deflection of the circularly polarized ray (which is superposition of a number of modes). This is connected with the reflection asymmetry for the circularly polarized rays. Indeed, reflection transformation changes the sign of the helicity. Maxwell equations are also not symmetrical with respect to the right and left circular polarizations [notice the opposite signs of gauge fields for right and left polarizations in Eqs. (19) and (21)]. Thus, these asymmetries cause topological spin transport under consideration.

Propagation of light in 2D photonic crystals has been considered in a recent paper. ${ }^{19}$ It was shown that a smooth inhomogeneity superimposed on the periodic crystal structure could induce topological spin transport of eigenmodes. In distinction from Ref. 19, we have shown that polarization transport of rays can exist in a perfectly periodic system itself.

To investigate in detail the dependence of the ray deflection on the angle of propagation $\alpha$ and on parameter $\beta$ (which characterizes the position of the ray with respect to the periodic lattice), numerical calculation of the integral in Eq. (26) with Eq. (33) had been carried out. Figure 2 shows the deflection $\delta z^{+}$of right-hand circularly polarized beam as a function of the angle of propagation $\alpha$ at two samples of different lengths $L$, with $\beta=0$. The existence of the well-
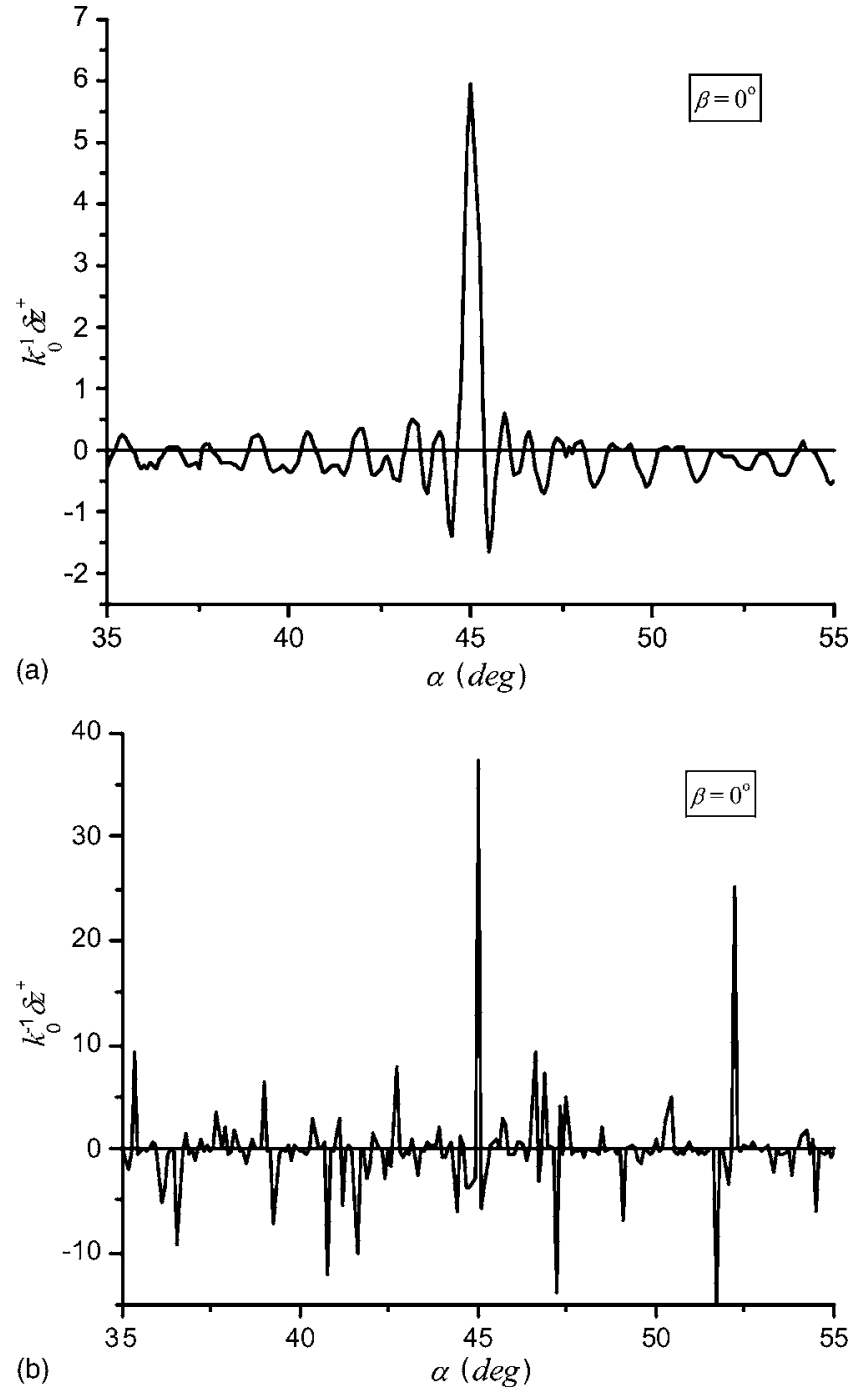

FIG. 2. Dependence of the deflection $\delta z^{+}$on the angle of propagation $\alpha$ for two samples of different sizes. (a) Sample of 40 periods of inhomogeneity. (b) Sample of 250 periods of inhomogeneity.

pronounced peak around $\alpha=\pi / 4$ can be understood if one recalls that the deflection is proportional to the (oriented) area bounded by the one-period ray trajectory in $\mathbf{p}$ space. The trajectory for $\alpha=\pi / 4$ is depicted in Fig. 1(a). If $\tan \alpha$ $=m($ or $\cot \alpha=m)$, where $m>1$ is an integer number [in Figs. 1 (b) and 1(c), $m=2$ and 3, respectively], the trajectories are closed lines, and the total oriented area of a one-period cycle equals zero, as well as Berry phase and ray deflection. At a longer sample [Fig. 2(b)], a number of resonant-like peaks at different angles $\alpha \neq \pi / 4$ arise. These peaks occur at angles that correspond to noncommensurable values of $p_{x 0}$ and $p_{y 0}$; i.e., to irrational values of $\tan \alpha$. That is why the picture has random-like structure. Actually, the noncommensurability of $p_{x 0}$ and $p_{y 0}$ gives rise to breaking of time-reversal symmetry (this is necessary for the Berry phase and accompanied phenomena) for the ray under consideration. Indeed, the corresponding trajectories for such rays in $\mathbf{p}$ space are open, and in general enclose nonzero oriented areas. The area changes its sign at time-reversal transformation. In samples of finite length, peaks occur at rational values of $\tan \alpha$ if $L$ is less than 

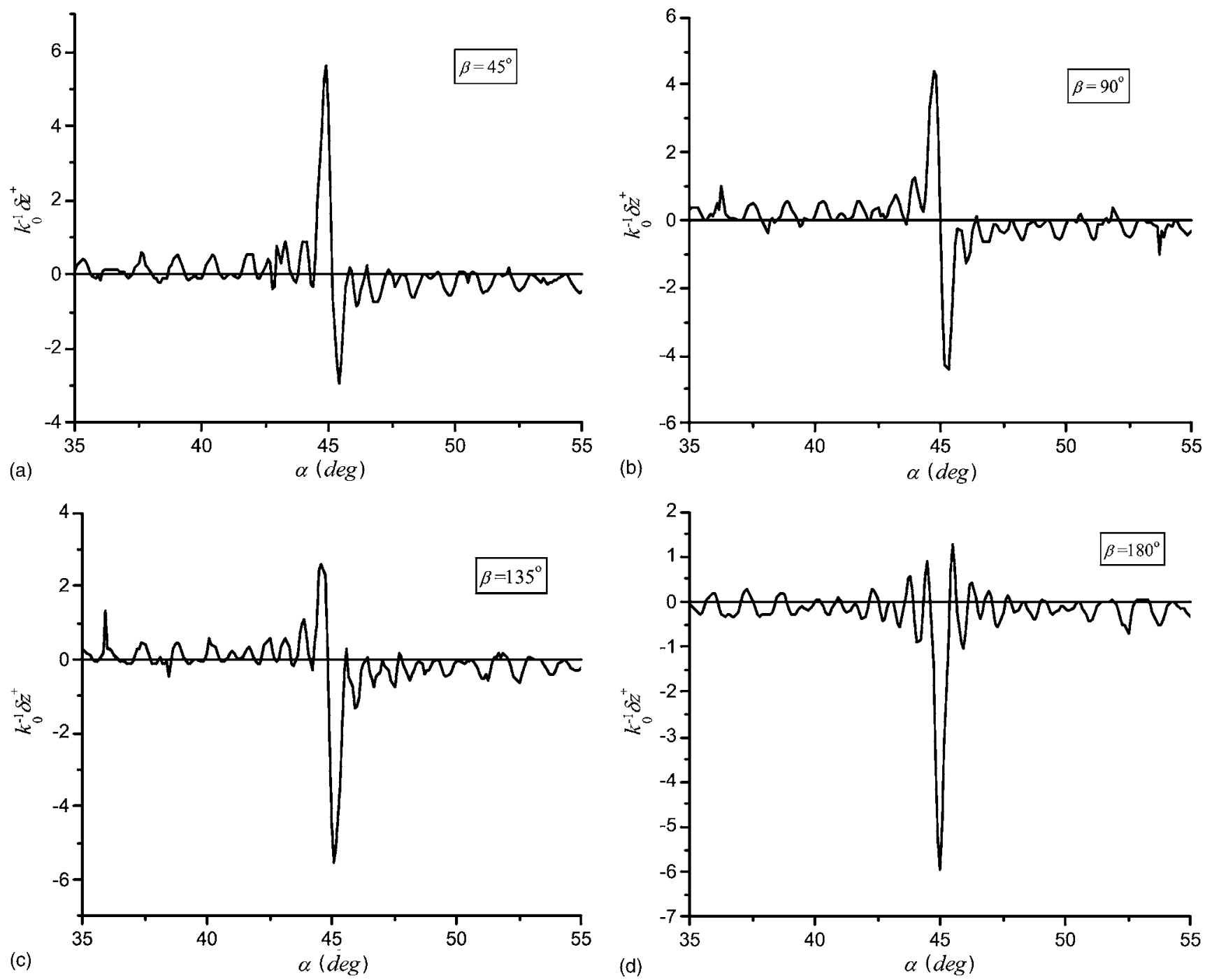

FIG. 3. Same as Fig. 2(a) for different $\beta$.

the period of wave trajectory in an infinite medium. This condition gives the following estimate for the characteristic width of the peaks:

$$
\delta \alpha \sim \frac{1}{\chi L} \ll 1 .
$$

In the limit $L \rightarrow \infty$, the peaks exist at irrational values of $\tan \alpha$. Amplitudes and widths of the peaks in Fig. 2 agree closely with Eq. (38) and estimate (39), respectively.

Figures 3(a)-3(d) present the dependences of the ray deflection $\delta z^{+}$on the angle of propagation $\alpha$ at different values of parameter $\beta$. The maximum value of $\delta z^{+}$decreases with increasing $\beta$ and changes sign at $\beta=\pi$. It should be noted that when $\beta=\pi / 2$, the deflection $\delta z^{ \pm}$vanishes at $\alpha=\pi / 4$, but occurs with the opposite signs just near the $\alpha=\pi / 4$. It can be also understood by considering the wave trajectory in $\mathbf{p}$ space. At $\beta=\pi / 2$ and $\alpha=\pi / 4$ it is a line (degenerated contour), and the zero area corresponds to it. However, when $\beta=\pi / 2$ and $\alpha=\pi / 4+\gamma$ with $|\gamma| \ll 1$, the trajectory is an open curve (for finite $L$ ) that is depicted qualitatively in Fig. 1(d). Obviously, it encloses a nonzero oriented area, and the sign of this area correlates with the sign of $\gamma$ [compare with Fig. 3(b)].

Finally, let us discuss the possibility of the experimental observation of the predicted effect. If, for example, $\delta=1 / 2$, Eq. (38) gives $\Delta z \equiv \delta z^{+}-\delta z^{-} \approx 10^{-1} \lambda L / L_{0}$ where $\lambda$ $=2 \pi / k_{0} n_{0}$, and $L_{0}=2 \pi / \chi$ is the period of the inhomogeneity. Hence, to get $\Delta z$ larger than the wavelength one needs a sample longer than ten inhomogeneity periods: $L>10 L_{0}$. [Note that the lengthening of the sample also leads, in accordance with Eq. (39), to the narrowing of the peak, and can make it indiscernible]. The splitting could be observed, for example, by using a converging (focused) beam whose width near the focal spot is of the order of a few wavelengths. When the splitting is too small to produce two separate beams of different polarization, the effect can be evidenced by measuring the polarization structure of the beam. Indeed, even small splitting of the "mathematical" ray of the linear polarization into two circularly polarized rays causes the appearance of the opposite-sign circular polarizations at the opposite sides of the initially linearly polarized physical beam. ${ }^{28}$ Another experimental possibility is to observe the propagation of a single circularly polarized beam, and to 
measure the deflection of its center of gravity, which can be done with an accuracy much higher than the width of the beam.

\section{CONCLUSION}

The first observation of the polarization transport of electromagnetic waves in isotropic inhomogeneous medium is due to Zel'dovich et al. ${ }^{14,15}$ Different theoretical explanations for this "optical Magnus effect" have been suggested based on the mode approach, ray approximation, and spinorbit interaction of photons. Recent theoretical investigations ${ }^{16-20}$ show that this effect has a pure topological nature and is closely related to the Berry phase. In Refs. 16 and 17, it has been demonstrated in the framework of the classical geometrical optics, while in Refs. 18 and 19 the gauge field approach was used. Gauge fields [Eqs. (21) and (22)] have been introduced in Refs. 18 and 19 out of a general reasoning, although without a full rigorous derivation. In Ref. 20 this field was obtained in a general form for relativistic quantum particles with arbitrary spin. Although the notion of a gauge field for photons was mentioned a number of times in papers dealing with Berry phase theory, a rigorous derivation of this field directly from Maxwell equations by general diagonalization and topological spin transport approach has been done in the present paper. It can be said that the results of Sec. II present a complete adiabatic and semiclassical theory of the topological spin transport of photons and provide a rigorous ground for the corresponding equations to be used.

To summarize, a semiclassical theory of the topological spin transport of photons in stationary inhomogeneous medium has been developed. The first step in the derivation is the diagonalization of the kinetic energy in Maxwell equations for monochromatic waves. A non-Abelian $\mathbf{U}(3)$ pure gauge potential in momentum space is induced, which in the adiabatic approximation breaks down into the product $\mathbf{U}(3)$ $\rightarrow \mathbf{U}(2) \times \mathbf{U}(1)$, where $\mathbf{U}(2)$ potential corresponds to transverse waves. Since photon is a massless particle, $\mathbf{U}(2)$ gauge potential is presented as a product of two Abelian potentials: $\mathbf{U}(2) \rightarrow \mathbf{U}(1) \times \mathbf{U}(1)$. These potentials describe the evolution of the right-hand and left-hand circularly polarized waves that are the photon spin eigenstates with helicities \pm 1 . The magnetic-monopole-like gauge field tensors corresponding to these potentials bring into existence a Lorentz-type force in the semiclassical equations of motion, and, hence, induce the topological spin transport of photons. As the result, the topological polarization ray deflection and splitting take place. ${ }^{16-18}$ It was mentioned earlier, ${ }^{18,19}$ that it is analogous to the spin and anomalous Hall effects for electrons in solids.

As an application of the developed theory the topological polarization transport of electromagnetic waves in a 3D medium with smooth periodic two-dimensional inhomogeneity is considered. By solving the geometrical optics ray equations perturbatively, we show that a circularly polarized ray located in the plane of inhomogeneity experiences the topological polarization deflection orthogonal to this plane. The deflections are of the opposite signs for the left-hand and right-hand polarized rays, and are extremely sensitive to the angle of propagation. Examples of the dependences of the deflection on the angle of propagation and on the position of the ray with respect to the periodic structure are presented, and the physical implications of the revealed features are discussed.

Experimental observation of the effects caused by the topological polarization transport of light presents a real challenge, because they could mix up with the diffractional broadening of the physical ray (beam), which is known to be of the same order of smallness; i.e., proportional to the wavelength. When this broadening is larger than the topological ray splitting the topological polarization transport can induce right-hand and left-hand circular polarizations at opposite sides of the single physical ray ${ }^{28}$ or can lead to the depolarization of the ray (compare with light depolarization that occurs due to the Berry phase increment ${ }^{29,30}$ ).

It should be also noted that the polarization deflection and splitting of rays are strongly connected with the Berry phase inherent in circularly polarized waves. Therefore our results can be used in studying the Berry phase in periodic structure. For example, predicted increase of Berry phase [Eqs. (36) and (37)] can be detected in two-dimensional periodic structures (photonic crystals) by measuring the rotation of the wave polarization plane.

\section{ACKNOWLEDGMENTS}

The work was partially supported by INTAS (Grant No. 03-55-1921), by Ukrainian President's Grant for Young Scientists GP/F8/51, and by the Israeli Science Foundation (Grant No. 328/02).
*Electronic address: k_bliokh@mail.ru

${ }^{1}$ M. V. Berry, Proc. R. Soc. London, Ser. A 392, 45 (1984).

${ }^{2}$ S. M. Rytov, Dokl. Akad. Nauk SSSR 18, 263 (1938) [C. R. (Dokl.) Acad. Sci. URSS 18, 263 (1938)]; reprinted in Topological Phases in Quantum Theory, edited by B. Markovski and S. I. Vinitsky (World Scientific, Singapore, 1989).

${ }^{3}$ V. V. Vladimirskii, Dokl. Akad. Nauk SSSR 31, 222 (1941); reprinted in Topological Phases in Quantum Theory, edited by B. Markovski and S. I. Vinitsky (World Scientific, Singapore,
1989).

${ }^{4}$ S. I. Vinnitski, V. L. Debrov, V. M. Dubovik, B. L. Markovski, and Yu. P. Stepanovskiy, Usp. Fiz. Nauk 160(6), 1 (1990) [Sov. Phys. Usp. 33, 403 (1990)].

${ }^{5}$ A. Tomita and R. Y. Chiao, Phys. Rev. Lett. 57, 937 (1986).

${ }^{6}$ M. Onoda and N. Nagaosa, J. Phys. Soc. Jpn. 71, 19 (2002).

${ }^{7}$ S. Murakami, N. Nagaosa, and S.-C. Zhang, Science 301, 1348 (2003).

${ }^{8}$ S. Murakami, cond-mat/0405003 (unpublished\}. 
${ }^{9}$ G. Sundaram and Q. Niu, Phys. Rev. B 59, 14915 (1999).

${ }^{10}$ T. Jungwirth, Q. Niu, and A. H. MacDonald, Phys. Rev. Lett. 88, 207208 (2002).

${ }^{11}$ D. Culcer, A. MacDonald, and Q. Niu, Phys. Rev. B 68, 045327 (2003).

${ }^{12}$ F. Zhou, Phys. Rev. B 70, 125321 (2004).

${ }^{13}$ K. Yu. Bliokh and Yu. P. Bliokh, quant-ph/0404144, Ann. Phys. (N.Y.) (to be published).

${ }^{14}$ A. V. Dooghin, N. D. Kundikova, V. S. Liberman, and B. Ya. Zel'dovich, Phys. Rev. A 45, 8204 (1992); B. Ya. Zel'dovich and V. S. Liberman, Kvantovaya Elektron. (Moscow) 17, 493 (1990) [Sov. J. Quantum Electron. 20, 427 (1990)]; A. V. Dooghin, B. Ya. Zel'dovich, N. D. Kundikova, and V. S. Liberman, Pis'ma Zh. Eksp. Teor. Fiz. 53, 186 (1991) [JETP Lett. 53, 197 (1991)]; A. V. Dooghin, B. Ya. Zel'dovich, N. D. Kundikova, and V. S. Liberman, Zh. Eksp. Teor. Fiz. 100, 1474 (1991) [Sov. Phys. JETP 73, 816 (1991)].

${ }^{15}$ V. S. Liberman and B. Ya. Zel'dovich, Phys. Rev. A 46, 5199 (1992).

${ }^{16}$ K. Yu. Bliokh and Yu. P. Bliokh, Pis'ma Zh. Eksp. Teor. Fiz. 79, 647 (2004) [JETP Lett. 79, 519 (2004)].

${ }^{17}$ K. Yu. Bliokh and Yu. P. Bliokh, Phys. Rev. E 70, 026605 (2004).

${ }^{18}$ K. Yu. Bliokh and Yu. P. Bliokh, Phys. Lett. A 333, 181 (2004); physics/0402110.
${ }^{19}$ M. Onoda, S. Murakami, and N. Nagaosa, Phys. Rev. Lett. 93, 083901 (2004).

${ }^{20}$ A. Bérard and H. Mohrbach, hep-th/0404165.

${ }^{21}$ A. I. Ahiezer and V. B. Berestetskii, Quantum Electrodynamics (Wiley, New York, 1965); V. B. Berestetskii, E. M. Lifshits, and L. P. Pitaevskii, Quantum Electrodynamics (Pergamon, Oxford, 1982).

${ }^{22}$ Yu. A. Kravtsov and Yu. I. Orlov, Geometrical Optics of Inhomogeneous Medium (Springer-Verlag, Berlin, 1990).

${ }^{23}$ K. Yu. Bliokh, J. Math. Phys. 43, 25 (2002); K. Yu. Bliokh, ibid. 43, 5624 (2002).

${ }^{24}$ F. Wilczek and A. Zee, Phys. Rev. Lett. 52, 2111 (1984).

${ }^{25}$ H.-Q. Zhou, S. Y. Cho, and R. H. McKenzie, Phys. Rev. Lett. 91, 186803 (2003).

${ }^{26}$ Zh. Fang et al., Science 302, 92 (2003).

${ }^{27}$ V. S. Liberman and B. Ya. Zel'dovich, Phys. Rev. E 49, 2389 (1994); A. Yu. Savchenko and B. Ya. Zel'dovich, ibid. 50, 2287 (1994); K. Yu. Bliokh and Yu. P. Stepanovskii, Zh. Eksp. Teor. Fiz. 124, 529 (2003) [JETP 97, 479 (2003)].

${ }^{28} \mathrm{~K}$. Yu. Bliokh and Yu. P. Bliokh (unpublished).

${ }^{29}$ A. C. Maggs and V. Rossetto, Phys. Rev. Lett. 87, 253901 (2001).

${ }^{30}$ D. Lacoste et al., Opt. Lett. 29, 2040 (2004). 